\title{
梳状-线性共聚物自组装的耗散粒子动力学模拟
}

\author{
王立权林嘉平* 张乾 \\ (华东理工大学 上海市先进聚合物材料重点实验室 材料科学与工程学院 上海 200237)
}

\begin{abstract}
摘要 梳状-线性共聚物在选择性溶剂中可以自组装形成两种不同类型的聚集体, 其中第 I 类的自组装发生在亲、疏溶 剂链之间, 而第 II 类发生在线性链和梳状亚结构之间. 本工作利用耗散粒子动力学方法, 分别研究了梳状-线性共聚物 在侧链和主链选择性溶剂中形成的这两类聚集体, 探讨了侧链长度和侧链数量等对聚集体类型及形貌的影响. 研究表 明, 第 II 类聚集体在侧链长度较短且侧链数量较多时容易形成. 将模拟结果与文献报道的实验结果相比较, 发现两者 能较好地吻合. 此外, 本研究获得了一些在实验中较难得到的信息, 有助于进一步理解梳状-线性共聚物的自组装行 为.
\end{abstract}

关键词 梳状-线性共聚物; 自组装; 耗散粒子动力学; 胶束; 相分离

\section{Dissipative Particle Dynamics Simulation on Self-Assembly of Comb-Coil Copolymers}

\author{
Wang, Liquan Lin, Jiaping* Zhang, Qian \\ (Shanghai Key Laboratory of Advanced Polymeric Materials, School of Materials Science and Engineering, East China \\ University of Science and Technology, Shanghai 200237)
}

\begin{abstract}
Using dissipative dynamics simulation, we studied self-assembly behavior of (A- $g$-B)- $b$-A comb-coil copolymers in selective solvents. The comb-coil copolymers, having two competitive length scales, are able to self-assemble into aggregates of different types, i.e., type I and type II. For the aggregates of type I, the phase separation occurs between the solvophobic and solvophilic blocks, which behave as asymmetric graft copolymers. While in the aggregates of type II, the phase separation takes place between coil and comb blocks, acting as diblock copolymers. The self-assembly of the comb-coil block copolymers in solvents selective to either graft arms or backbone was investigated. The effects of the number and length of graft arms on the self-assembly behavior were examined. In the solvents selective to graft arms, the comb-coil copolymers tend to assemble into spherical micelles of type II, where the comb and coil blocks form the shell and core, respectively. This is due to the fact that the crowd of the comb blocks in the shell can be alleviated by forming high-curvature structures. In addition, such a crowd can also be alleviated by decreasing the length of graft arms and therefore, vesicles were observed when the graft arms are short. In addition, the decrease in the interaction strength between backbone and graft arms (and solvents) favors the formation of the aggregates of type II. In the solvents selective to backbones, the comb-coil copolymers incline to form low-curvature aggregates of type II, such as disklike micelles and vesicles. By forming low-curvature structures, the rod-like comb blocks can be tightly packed in the cores of the aggregates. When the comb-coil copolymers form the aggregates of type I in both solvents, the morphologies are very sensitive to the length of the graft arms. For example, in solvents selective to graft arms, as the length of graft arms increases, a morphological transformation of large-compound micelle $\rightarrow$ vesicle $\rightarrow$ cylindrical micelle $\rightarrow$ spherical micelle was observed. The simulation results were compared with the available experimental findings reported in the literatures, and an agreement was observed. In addition, the simulations predict some behaviors that have not been observed yet. The present work is helpful for further understanding the competitive self-assembly behavior of the comb-coil copolymers.

Keywords comb-coil copolymer; self-assembly; dissipative particle dynamics simulation; micelle; phase separation
\end{abstract}

\section{1 引言}

高分子自组装是超分子化学的重要组成部分，是高 分子研究领域的热点之一 ${ }^{[1 ~ 9]}$. 其中, 研究得最为深入 的当属共聚物在溶液中的胶束化. 通过自组装, 共聚物 可以在选择性溶剂中形成球形胶束、棒状胶束、囊泡以
及它们的共存体 ${ }^{[10,11]}$. 聚集体的形貌受制样过程、温度、 溶剂选择性和链段长度等因素的影响.

在共聚物自组装中, 与溶剂相溶的链段通常形成聚 集体的壳，而不相溶的链段则形成核(第 I 类). 这是传统 的聚集体成核成壳机制，即组分间的相分离. 然而，一

* E-mail: jlin@ecust.edu.cn; Tel.: 021-64253370

Received January 20, 2013; published April 23, 2013.

Supporting information for this article is available free of charge via the Internet at http://sioc-journal.cn.

Project supported by the National Natural Science Foundation of China (Nos. 50925308, 21234002).

项目受国家自然科学基金(Nos. 50925308, 21234002)资助. 本文由张希院士约稿. 
些具有复杂结构的共聚物却呈现出不同于传统成核成 壳机制的自组装行为. 典型的例子如梳状-线性共聚物 的自组装(见图 1a) ${ }^{[12 \sim 18]}$. 该共聚物可以发生两种尺度的 相分离: 一是侧链与主链间的相分离, 表现出不对称接 枝共聚物的特性(第 I 类, 见图 $1 \mathrm{~b}$ ); 二是线性链段与梳 状亚结构之间的相分离, 表现出类似于不对称二嵌段共 聚物的特性(第 II 类, 见图 1c). 在第 II 类相分离中, 与 溶剂相溶(或不溶)的链段可以出现在聚集体的核(或壳) 中(注：图 1 仅例示了在侧链选择性溶剂的自组装).

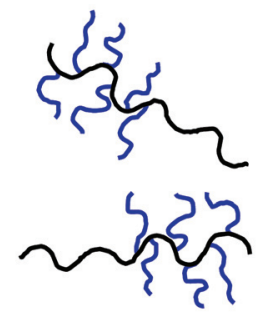

(a) (b)

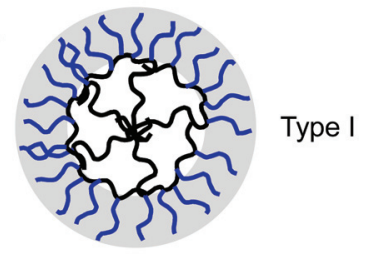

(c)

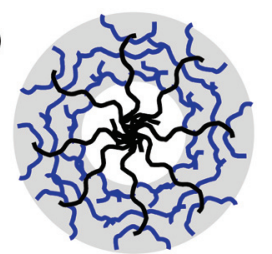

Type II
图 1 由(a)梳状-线性共聚物侧链选择性溶剂中自组装形成的(b)第 I 类和(c)第 II 类聚集体的示意图

Figure 1 Sketch of the aggregates of types I (b) and II (c) formed by comb-coil copolymers (a) in solvents selective to graft arms

近年来，随着聚合技术的进步，研究者合成了一系 列梳状一线性共聚物, 并在自组装研究中观测到了第 II 类聚集体结构 ${ }^{[19 ~ 23]}$. 邱等 ${ }^{[24]}$ 研究了(聚苯乙烯- $g$-聚异戊 二烯)- $b$-聚苯乙烯((PS- $g$-PI)- $b$-PS)共聚物在正烷烃中的 自组装行为. 他们发现, 胶束的尺寸呈现出明显的双分 布现象. 这是第 I 类和第 II 类聚集体共存的结果. 此外, 他们初步地研究了溶剂选择性、接枝度和侧链长度对自 组装行为的影响, 研究表明增加溶剂选择性或接枝度及 侧链长度有利于两类聚集体的形成和对应分布峰的分 离. 王等 ${ }^{[25]}$ 利用自洽场理论计算了单胶束体系的自由 能, 并结合热力学方法分析了由梳状-线性共聚物形成 的这两类聚集体. 结果表明, 在这两类聚集体中, 通常 一类是稳态结构, 而另一类是亚稳态结构. 这与链段的 长度和溶剂选择性密切相关. 这些研究对于理解梳状线性共聚物的自组装行为起到了重要的作用. 然而, 梳 状-线性共聚物分子结构对其自组装行为的具体影响规 律尚不太明确, 比如侧链长度对第 I 类和第 II 类聚集体 的形成条件的影响等. 为了全面掌握这类聚合物的自组 装行为, 相关工作还有待开展.

计算机模拟可以被用来系统地研究梳状-线性共聚 物的自组装行为, 以获得实验上较难观测到的结果. 耗 散粒子动力学(DPD) 是一种模拟复杂流体的介观方法, 最早由 Hoogerbrugge 和 Koelman 提出 ${ }^{[26,27]}$, 后经 Groot
等完善而沿用至今 ${ }^{28,29]}$. 目前，该方法已被广泛用来研 究聚合物体系, 如共聚物薄膜和共聚物溶液等 ${ }^{[30 ~ 35]}$. 比 如, 蒋等 ${ }^{[34]}$ 用 DPD 研究了三嵌段共聚物的自组装行为, 观测到了与实验现象一致的同心圆和车厘子状聚集体, 并预测了它们的形成条件. 为了进一步理解和掌握梳 状一线性共聚物的自组装行为, 本文将采用上述方法, 模拟梳状一线性共聚物在选择性溶剂中的自组装行为, 并探讨侧链长度和侧链数量等因素对聚集体结构的影 响.

\section{2 结果与讨论}

在模拟中，首先构建了如图 2 所示的梳状-线性共 聚物, 其中侧链(graft arm)均匀地接枝在主链(backbone) 上. 该梳状一线性共聚物仅含有两种组分. 为了区分线 性链段(coil block)和梳状部分(comb block)的主链, 两个 链段的粒子分别用绿色和红色表示(注：它们具有相同 化学性质). 在下文中, 链段的颜色与图 2 对应, 将不再 累述. 研究内容分两部分: 一部分是梳状-线性共聚物 在侧链选择性溶剂中的自组装, 而另一部分是梳状一线 性共聚物在主链(或线性链段)选择性溶剂中的自组装.

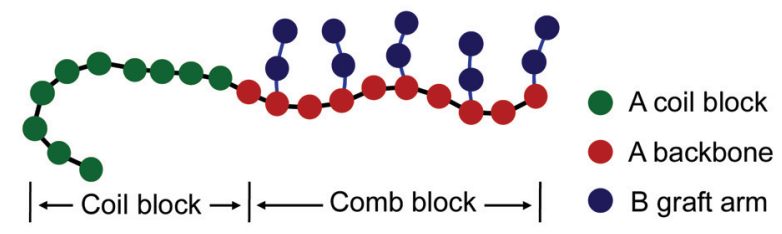

图 2 梳状-线性共聚物的 DPD 模型. 虽然线性链段和梳状结构的主 链用不同颜色的珠子表示, 但它们具有相同的化学性质

Figure 2 DPD model of a comb-coil copolymer. The coil block and the backbone of comb block, having the same chemical nature, are represented as green and red beads, respectively

\section{1 在侧链选择性溶剂中的自组装}

图 3 是由梳状一线性共聚物在侧链选择性溶剂中形 成的两类典型的聚集体。这两类聚集体分别由 $\left(\mathrm{A}_{10}-g-\left(\mathrm{B}_{2}\right)_{10}\right)-b-\mathrm{A}_{20}$ 和 $\left(\mathrm{A}_{10}-g-\left(\mathrm{B}_{4}\right)_{5}\right)-b-\mathrm{A}_{20}$ 共聚物自组装 形成(注: $\left(\mathrm{A}_{k}-g-\left(\mathrm{B}_{y}\right)_{x}\right)-b-\mathrm{A}_{z}$ 中的 $x, y, z$ 和 $k$ 分别表示侧链 数量、每条侧链粒子数、线性链段粒子数和主链粒子数). 在两个体系中, $\mathrm{A}$ (或 B)组分具有相同的质量分数. 然而, 它们却自组装形成不同类型的聚集体. $\left(\mathrm{A}_{10}-g-\left(\mathrm{B}_{2}\right)_{10}\right)-$ $b-\mathrm{A}_{20}$ 共聚物形成如图 3a 所示的第 II 类聚集体, 即以 $\mathrm{A}_{20}$ 线性链段为核、 $\mathrm{A}_{10^{-}} g-\left(\mathrm{B}_{2}\right)_{10}$ 梳状亚结构为壳的球形胶束. 这可以从图 $3 \mathrm{~b}$ 所示的径向密度分布中辨识, 其中 $\mathrm{A}_{10}$ 主 链和 $\mathrm{B}$ 侧链具有相似的分布, 最大密度分布处几乎重叠. 这说明 $\mathrm{A}_{10}$ 主链和 $\mathrm{B}$ 侧链都形成了球形胶束的壳. 不同 于 $\left(\mathrm{A}_{10}-g-\left(\mathrm{B}_{2}\right)_{10}\right)-b-\mathrm{A}_{20}$ 共聚物, $\left(\mathrm{A}_{10}-g-\left(\mathrm{B}_{4}\right)_{5}\right)-b-\mathrm{A}_{20}$ 共聚物 形成了如图 $3 \mathrm{c}$ 所示的第 $\mathrm{I}$ 类聚集体, 即以 $\mathrm{A}$ 为核、侧链 为壳的球形胶束. 由图 $3 \mathrm{~d}$ 的可知, $\mathrm{A}_{10}$ 主链与 $\mathrm{B}$ 侧链的 分布发生偏移, 其中 $\mathrm{A}_{10}$ 主链主要分布在核/壳界面上. 
第 I 类和第 II 类聚集体的聚合物链个数分别为 $25 \pm 4$ 和 $28 \pm 3$. (a)

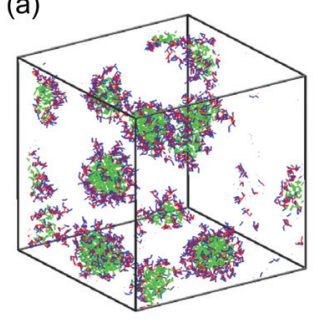

(c)

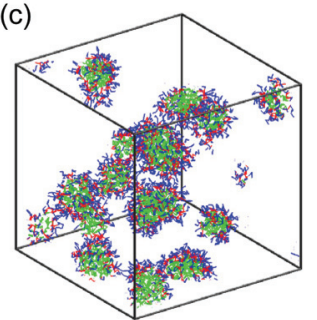

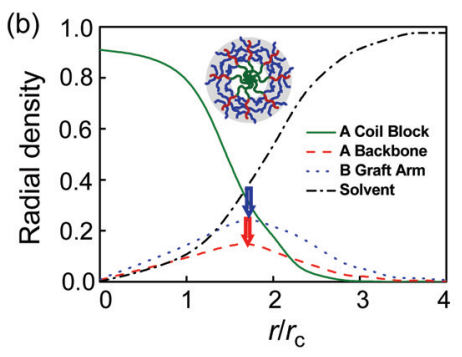

(d)

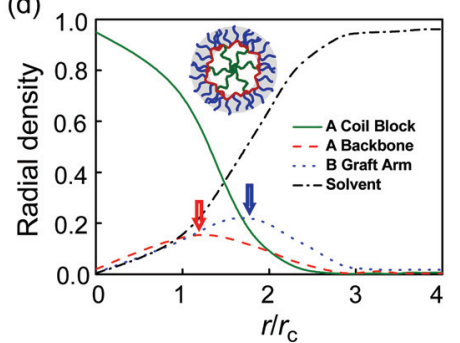

图 3 由( $\mathrm{a}, \mathrm{b})\left(\mathrm{A}_{10}-g-\left(\mathrm{B}_{2}\right)_{10}\right)-b-\mathrm{A}_{20}$ 和 $(\mathrm{c}, \mathrm{d})\left(\mathrm{A}_{10}-g-\left(\mathrm{B}_{4}\right)_{5}\right)-b-\mathrm{A}_{20}$ 梳状-线性 共聚物形成的胶束及其径向密度分布. 在 $(b, d)$ 中, 蓝色和红色箭头所 指为侧链和主链的最大密度分布处; 横坐标原点对应于胶束的中心; 图内的示意图为相应的胶束结构. 相互作用参数为 $a_{\mathrm{AS}}=a_{\mathrm{AB}}=50$

Figure 3 Micelles self-assembled from (a) $\left(\mathrm{A}_{10}-g-\left(\mathrm{B}_{2}\right)_{10}\right)-b-\mathrm{A}_{20}$ and (c) $\left(\mathrm{A}_{10}-g-\left(\mathrm{B}_{4}\right)_{5}\right)-b-\mathrm{A}_{20}$ comb-coil copolymers. $(\mathrm{b}, \mathrm{d})$ shows the corresponding radial density distributions, and the inner schematics are the structures of the micelles. The blue and red arrows indicate the maximum densities of the graft arms and backbone, respectively. The interaction parameters are $a_{\mathrm{AS}}=a_{\mathrm{AB}}=50$

值得注意的是, 利用密度分布区分第 I 类和第 II 类 聚集体存在着很多困难(注：计算方法见 “支持信息 (Supporting Information)” 的第 1 部分). 第一、空间网格 的精度对密度分布影响很大. 如果网格过细, 那么密度 分布离散而不光滑, 无法描绘密度分布的总体趋势; 如 果网格过粗, 那么密度分布的细节有可能被忽略, 以致 无法区分第 I 类和第 II 类聚集体. 因此, 网格精度需优 化. 第二、中心点的选取也会影响第 I 类和第 II 类聚集 体的判断. 由于本文以聚集体核的重心替代中心点, 因 此只有当聚集体的几何形状比较规则(如球形)时才适用. 当聚集体形状不规则时, 我们可用指定方向上的密度分 布替代径向密度分布. 这两类聚集体还可以通过计算主 链末端距矢量与界面法线之间的夹角来区分第 $\mathrm{I}$ 类和第 II 类聚集体(详见“支持信息”的第 2 部分). 研究发现, 在 第 I 类聚集体中, 约 $60 \%$ 的角是大于 $\pi / 2$; 在第 II 类聚集 体中, 约 $73 \%$ 的角度小于 $\pi / 2$. 这是由于主链分布在第 I 类聚集体的核内、第 II 类聚集体的核外. 如果以 $50 \%$ 作 为区分点, 那么可以较为定量地区分第 I 类和第 II 类聚 集体. 我们发现, “角度法” 和密度分布法的判定结果 是一致的. 与密度分布法相比, “角度法” 不受网格精 度的限制, 能较方便地应用于不规则聚集体类型的判 定.
由图 3 可知, 梳状-线性共聚物的分子结构对聚集 体的类型具有重要的影响. 通过研究 $\left(\mathrm{A}_{10}-g-\left(\mathrm{B}_{y}\right)_{x}\right)-b-\mathrm{A}_{20}$ 共聚物在不同侧链数量 $x$ 和侧链长度 $y$ 下的自组装行为, 总结了如图 4 所示的聚集体稳定区域图. 图 4a 和图 $4 \mathrm{~b}$ 分别对应于不同的相互作用参数 $a_{\mathrm{AS}}$ 和 $a_{\mathrm{AB}}$. 图中的黄 色和蓝色区域分别对应于第 I 和 II 类聚集体的稳定区域. 由图 4 可知，第 II 类聚集体的形成条件较为苛刻: 只有 在侧链数量足够多(即接枝度足够高)和侧链较短时才能 形成. 此外, 第 II 类聚集体的结构对侧链长度的变化不 敏感，趋向于形成球形胶束，只有当侧链长度非常短时 $(y=1)$ 才形成囊泡(vesicle). 与第 II 类聚集体相比，第 I 类聚集体的结构受侧链长度的影响较大. 比如, 对于 $x=3$ 的共聚物, 随着侧链长度的增加, 聚集体结构发生 从复合胶束(large-compound micelle)到囊泡到棒状胶束 (cylindrical micelle)再到球状胶束(spherical micelle)的转 变(如图 $4 \mathrm{~b}$ 所示). 该转变趋势与二嵌段共聚物在组分变 化下的形貌转变趋势是一样的(注：侧链长度增加等效 于 $\mathrm{B}$ 组分增加). 通过比较图 $4 \mathrm{a}$ 和图 $4 \mathrm{~b}$ 发现, 降低 $a_{\mathrm{AS}}$ 和 $a_{\mathrm{AB}}$ 使得第 II 类聚集体的区域(蓝色)明显增大. 这表 明降低 $a_{\mathrm{AS}}$ 和 $a_{\mathrm{AB}}$ 有利于第 II 类聚集体的形成. (注: 由 于所用模型要求侧链在主链上等距分布, 因此模拟的数 据点较为稀疏.)
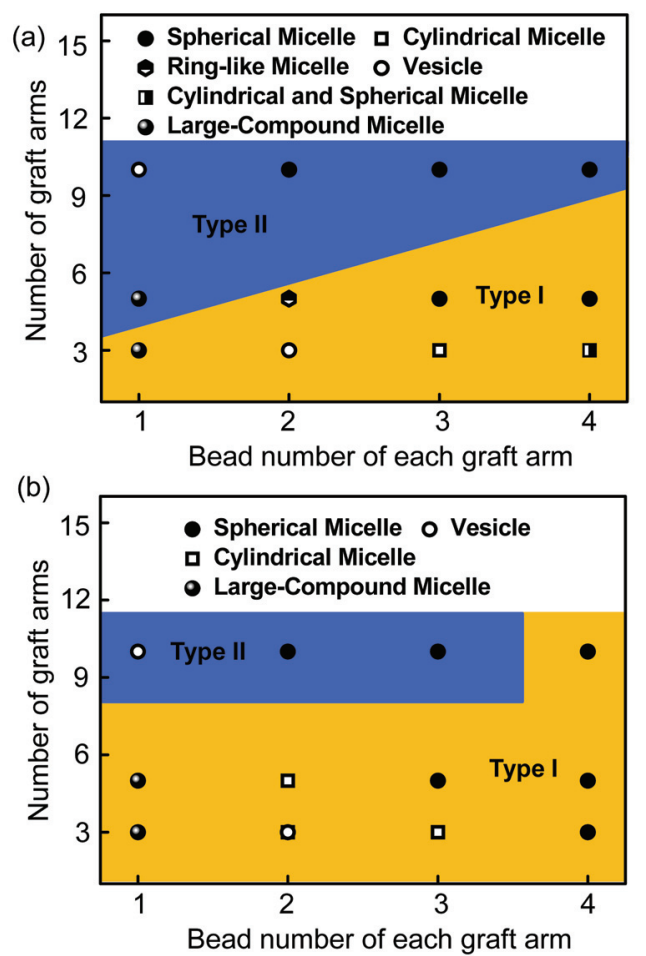

图 4 具有不同侧链数量 $x$ 和侧链长度 $y$ 的 $\left(\mathrm{A}_{10}-g-\left(\mathrm{B}_{y}\right)_{x}\right)-b-\mathrm{A}_{20}$ 共聚物 的聚集体稳定区域图: (a) $a_{\mathrm{AS}}=a_{\mathrm{AB}}=50$ 和(b) $a_{\mathrm{AS}}=a_{\mathrm{AB}}=80$. 图中黄色 和蓝色区域分别对应于第 I 和 II 类聚集体的稳定区域

Figure 4 Morphological region in the plane of number $x$ of graft arms vs. bead number $y$ of each graft arm for $\left(\mathrm{A}_{10}-g-\left(\mathrm{B}_{y}\right)_{x}\right)-b$ - $\mathrm{A}_{20}$ comb-coil copolymers with (a) $a_{\mathrm{AS}}=a_{\mathrm{AB}}=50$ and (b) $a_{\mathrm{AS}}=a_{\mathrm{AB}}=80$. The yellow and blue regions correspond to the regions of type I and type II 
第 II 类聚集体的形貌不但受侧链长度的影响较小, 而且受线性链段的长度影响也很小. 图 5 是 $\left(\mathrm{A}_{10}-g-\left(\mathrm{B}_{2}\right)_{10}\right)-b-\mathrm{A}_{z}$ 共聚物在不同线性链段长度 $z$ 下形成 的聚集体形貌. 由图可见, 在 $z=15 \sim 60$ 的变化范围内, 该共聚物仅形成一种形貌的聚集体, 即球形胶束. 这说 明，当梳状一线性共聚物在侧链选择性溶剂中形成第 II 类聚集体时, 球形结构是非常有利的. 可能的原因如下: 在第 II 类聚集体中, 梳状亚结构在壳上的特殊分布会造 成界面的局部拥挤, 而形成高曲率的球形结构有利于缓 解这种拥挤. 此外, 当侧链非常短时, 该拥挤现象也能 得到缓解, 因而能形成低曲率的囊泡(见图 4, $y=1$ 且 $x$ $=10)$.

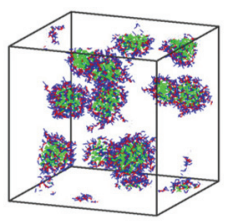

(a)

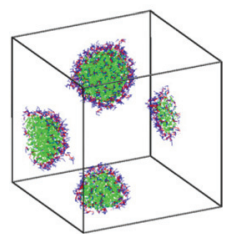

(d)

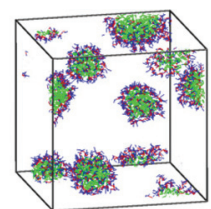

(b)

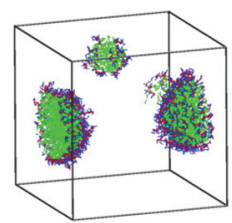

(e)

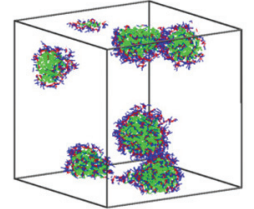

(c)

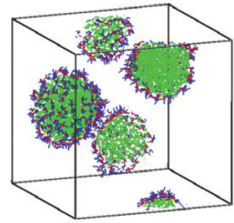

(f)
图 5 由 $\left(\mathrm{A}_{10}-g-\left(\mathrm{B}_{2}\right)_{10}\right)-b-\mathrm{A}_{z}$ 共聚物在不同线性链段长度下形成的第 II 类聚集体: (a) $z=15$; (b) $z=25$; (c) $z=30$; (d) $z=40$; (e) $z=50$; (f) $z=60$. 相互作用参数为 $a_{\mathrm{AS}}=a_{\mathrm{AB}}=50$

Figure 5 Micelles (type II) self-assembled from $\mathrm{A}_{z}-b-\left(\mathrm{A}_{10}-g-\left(\mathrm{B}_{2}\right)_{10}\right)$ comb-coil copolymers. The length of coil blocks are (a) $z=15$, (b) $z=25$, (c) $z=30$, (d) $z=40$, (e) $z=50$, and (f) $z=60$, respectively. The interaction parameters are $a_{\mathrm{AS}}=a_{\mathrm{AB}}=50$

最近, 邱等 ${ }^{[24]}$ 研究了(PS- $g$-PI)- $b$-PS 梳状-线性共聚 物在正烷烃中的自组装行为, 其中正烷烃是侧链 PI 的 选择性溶剂. 研究发现, 该共聚物自组装形成两类胶束, 即第 I 和 II 类聚集体. 接枝度最高的共聚物形成较多的 第 II 类聚集体, 而接枝度中等或偏低的共聚物形成少量 的第 II 类聚集体(见参考文献[24]的图 6). 这与我们的发 现是一致的, 即只有当接枝度足够高时, 梳状-线性共 聚物才形成第 II 类聚集体.

此外, 他们还研究了溶剂选择性对聚集体结构的影 响. 研究发现, 随着烷烃链碳原子数量的增加, 即正庚 烷 $\rightarrow$ 正癸烷 $\rightarrow$ 正十二烷，第 II 类聚集体的比例有所上升. 其中, PI 的溶解性随着碳元素比例的增加而增加. 这说 明, 梳状-线性共聚物溶解性的增强有利于第 II 类聚集 体的形成. 鉴于篇幅限制, 本研究并没有直接研究溶剂 选择性对聚集体结构的影响. 然而, 通过对照图 4a 和图 $4 \mathrm{~b}$ 可知, $\left(\mathrm{A}_{10}-g-\left(\mathrm{B}_{4}\right)_{10}\right)-b-\mathrm{A}_{20}$ 共聚物在较大的 $a_{\mathrm{AS}}$ 和 $a_{\mathrm{AB}}$ 下形成第 I 类聚集体, 而在较小的 $a_{\mathrm{AS}}$ 和 $a_{\mathrm{AB}}$ 下形成第 II 类聚集体. 虽然 $a_{\mathrm{AS}}$ 减小表示的是疏溶剂链段溶解性的
下降(并不是亲溶剂链段溶剂性的增强), 但是这从侧面 反映出共聚物溶解性的增强有利于第 II 类聚集体的形 成. 这一结果从一定程度上佐证了邱等发现的实验现 象.

值得注意的是，他们所报道的聚集体是两类聚集体 的共存体, 而在我们前面的研究中, 并未观测到这一现 象. 对于这一结果, 最为可能的原因是: 在我们的参数 条件下，第 I 和 II 类聚集体的能差和势垒较大，因而其 中一类聚集体的形成占有绝对的优势 ${ }^{[25]}$. 由于 $\left(\mathrm{A}_{10}-g-\left(\mathrm{B}_{4}\right)_{10}\right)-b-\mathrm{A}_{20}$ 共聚物在不同的 $a_{\mathrm{AS}}$ 和 $a_{\mathrm{AB}}$ 下可以形 成不同类型的聚集体, 因此通过改变 $a_{\mathrm{AS}}$ 和 $a_{\mathrm{AB}}$ 就有可 能观测到两类聚集体共存的现象. 图 6 所示的是该共聚 物在 $a_{\mathrm{AS}}=80$ 和 $a_{\mathrm{AB}}=50$ 时形成的聚集体. 由图 $6 \mathrm{a}$ 可知, 第 I 和 II 类聚集体同时存在于一个体系中. 这可以从图 $6 \mathrm{~b}$ 中辨识, 较大的核对应于第 II 类聚集体, 而较小的核 对应于第 I 类聚集体. 共存体系的形成是由于这两类胶 束在该参数下的能差和势垒较小.

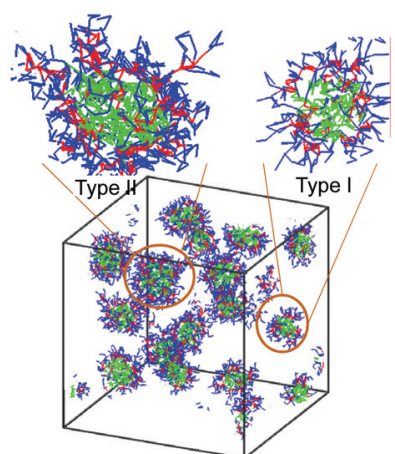

(a)

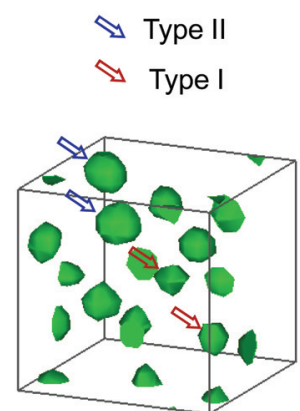

(b)
图 6 由 $\left(\mathrm{A}_{10}-g-\left(\mathrm{B}_{4}\right)_{10}\right)-b-\mathrm{A}_{20}$ 共聚物形成的(a)聚集体及其(b)相应的三 维密度分布(仅线性链段), 其中第 I 和 II 类聚集体共存. 在(a)中, 顶部 所示为单个的第 I 和 II 类聚集体的放大图; 在(b)中, 红色和蓝色箭头 所指的分别为第 $\mathrm{I}$ 和 II 类聚集体. 相互作用参数为 $a_{\mathrm{AB}}=50$ 和 $a_{\mathrm{AS}}=80$ Figure 6 (a) Micelles self-assembled from $\left(\mathrm{A}_{10}-g-\left(\mathrm{B}_{4}\right)_{10}\right)-b-\mathrm{A}_{20}$ comb-coil block copolymers, where the type I and II coexist. The top views are the magnification of the micelle of type I and II. (b) Three-dimensional density of the coil blocks. The red and blue arrows indicate the micelles of type I and II, respectively. The interaction parameters are $a_{\mathrm{AB}}=50$ and $a_{\mathrm{AS}}=80$

\section{2 在主链选择性溶剂中的自组装}

在主链选择性溶剂中, 梳状一线性共聚物也能形成 第 I 和 II 类聚集体. 图 7 是分别由 $\left(\mathrm{A}_{10}-g-\left(\mathrm{B}_{8}\right)_{5}\right)-b-\mathrm{A}_{10}$ 和 $\left(\mathrm{A}_{10}-g-\left(\mathrm{B}_{4}\right)_{10}\right)-b-\mathrm{A}_{10}$ 共聚物形成的第 I 和 II 类聚集体. 图 $7 \mathrm{a}$ 为一个典型的囊泡结构, 其中囊泡壁由疏溶剂链 段 $\mathrm{B}$ 形成, 而囊泡壳由亲溶剂链段 $\mathrm{A}$ 形成. 从图 $7 \mathrm{~b}$ 中 可知, 梳状部分的主链主要分布在囊泡的亲/疏界面附 近，而侧链主要分布在囊泡壁的中心. 这说明梳状结构 的主链和侧链是分离的, 此时形成了第 I 类聚集体. 图 $7 \mathrm{c}$ 是一个典型的盘状胶束. 从图 $7 \mathrm{~d}$ 中的密度分布可知, 梳状部分的主链与侧链均匀分布在胶束的核内，而线性 
链段分布在壳层. 这表明了第 II 类聚集体的形成, 即以 梳状亚结构为核、线性链段为壳的盘状胶束. 虽然 $\left(\mathrm{A}_{10}-g-\left(\mathrm{B}_{8}\right)_{5}\right)-b-\mathrm{A}_{10}$ 和 $\left(\mathrm{A}_{10}-g-\left(\mathrm{B}_{4}\right)_{10}\right)-b-\mathrm{A}_{10}$ 共聚物具有质 量分数相同的 $\mathrm{B}$ (或 $\mathrm{A}$ )组分, 但是它们却形成了两类截 然不同的结构. 这说明, 分子结构对于梳状-线性共聚 物在主链选择性溶剂中形成的聚集体也具有重要的影 响. (a)

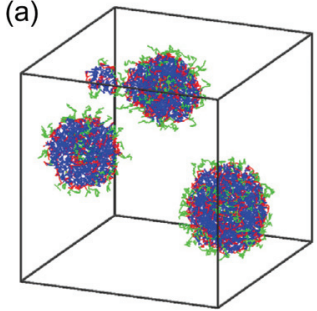

(c)

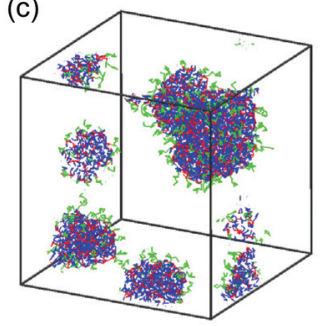

(b)

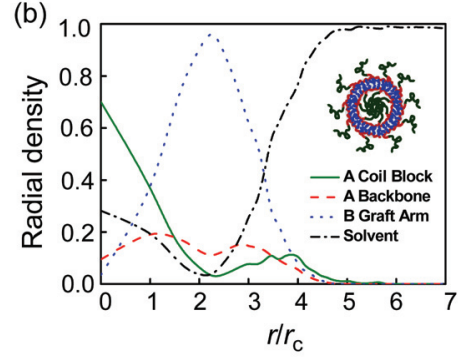

(d)

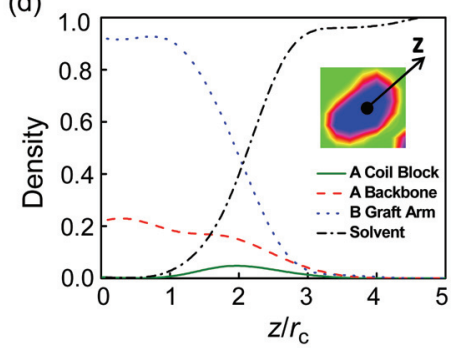

图 7 由 $(\mathrm{a}, \mathrm{b})\left(\mathrm{A}_{10}-g-\left(\mathrm{B}_{8}\right)_{5}\right)-b-\mathrm{A}_{10}$ 和(c, d) $\left(\mathrm{A}_{10}-g-\left(\mathrm{B}_{4}\right)_{10}\right)-b-\mathrm{A}_{10}$ 梳状-线 性共聚物形成的聚集体及其密度分布. 其中, (b)和(d)分别为囊泡的径 向密度分布图和盘状胶束在 $z$ 方向上的密度分布. (b)内的示意图为囊 泡. 相互作用参数为 $a_{\mathrm{AB}}=a_{\mathrm{BS}}=50$

Figure 7 Aggregates self-assembled from (a) $\left(\mathrm{A}_{10^{-}} g-\left(\mathrm{B}_{8}\right)_{5}\right)-b-\mathrm{A}_{10}$ and (c) $\left(\mathrm{A}_{10}-\mathrm{g}-\left(\mathrm{B}_{4}\right)_{10}\right)-b-\mathrm{A}_{10}$ comb-coil copolymers. (b) and (d) shows the radial density distribution of the vesicle and the density profile along $z$ direction of the disk-like micelle. Schematic in the inner (b) shows a vesicle. The interaction parameters are $a_{\mathrm{AB}}=a_{\mathrm{BS}}=50$

图 8 是由梳状一线性共聚物在主链选择性溶剂中形 成的聚集体的稳定区域图. 与在侧链选择性溶剂中的情 况类似, 第 II 类聚集体在侧链数量较多(接枝度较高)和 侧链较短时容易形成. 在第 II 类聚集体中, 梳状-线性 共聚物趋于形成盘状胶束(disklike micelle), 而当侧链较 长时可形成囊泡. 一个重要的原因是: 在形成第 II 类聚 集体时, 梳状的亚结构类似于增粗的刚性链段 ${ }^{[19,20]}$, 而 低曲率的盘状胶束(或囊泡)的形成可以保证梳状亚结构 在聚集体核内的紧密堆积 ${ }^{[36]}$. 与第 II 类聚集体相比, 第 I 类聚集体的形貌较为丰富. 例如, 当侧链数量 $x=3$ 时, 随着侧链增长, 聚集体形貌发生从无序(disorder)到胶束 再到双分子膜(bilayer)的转变.

目前，虽然没有直接的实验数据来支持这一模拟结 果, 但是可以与一些相关的文献报道进行比较. 刘等 ${ }^{[22]}$ 合成了线性-梳状-线性(coil-comb-coil)的三嵌段共聚物. 研究发现, 该共聚物可以形成以梳状部分为核、两个线 性链段为壳的球形复合胶束. 该胶束就是本研究所发现
的第 II 类聚集体，这从侧面证实了梳状-线性共聚物可 形成第 II 类聚集体.

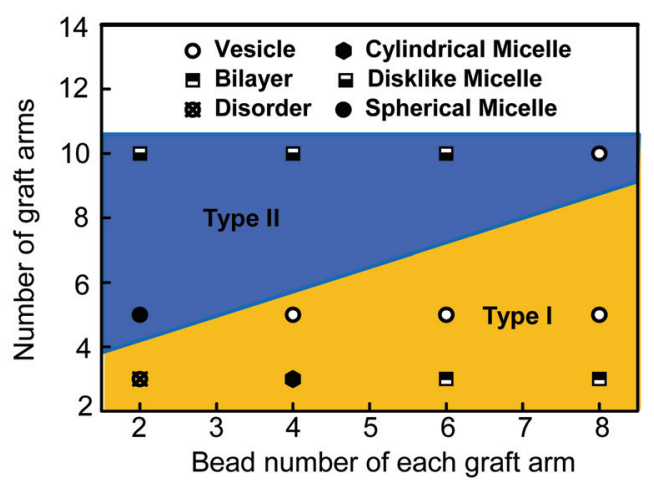

图 8 具有不同侧链数量 $x$ 和侧链长度 $y$ 的 $\left(\mathrm{A}_{10}-g-\left(\mathrm{B}_{y}\right)_{x}\right)-b-\mathrm{A}_{10}$ 共聚物 的聚集体稳定区域图, 其中 $a_{\mathrm{AB}}=a_{\mathrm{BS}}=50$. 图中黄色和蓝色区域分别 对应于第 I 和 II 类聚集体的稳定区域

Figure 8 Morphological region in the plane of number $x$ of graft arms vs. bead number $y$ of each graft arm for $\left(\mathrm{A}_{10}-g-\left(\mathrm{B}_{y}\right)_{x}\right)-b-\mathrm{A}_{10}$ comb-coil copolymers with $a_{\mathrm{AB}}=a_{\mathrm{BS}}=50$. The yellow and blue regions correspond to the regions of type I and type II

上述结果表明，无论是在侧链还是主链选择性溶剂 中, 梳状-线性共聚物都能自组装形成第 I 类和第 II 类聚 集体. 其中, 第 II 类聚集体的形貌较为单一, 且具有一 定的应用潜力. 比如, 它可以作为药物控释的载体, 其 原因有二. 第一、用第 II 类聚集体包裹小分子物质(如 纳米粒子和药物等)将具有较高的包封率. 这是由于梳 状亚结构在壳上的拥挤分布将造成核内线性链段的高 度受挫，而小分子物质的加入有利于缓解这类受挫. 第 二、由梳状亚结构形成的特殊壳层将阻碍小分子物质 的释放, 能起到缓释的效果. 希望本研究对于理解梳 状一线性共聚物的自组装行为具有一定的指导意义.

\section{3 结论}

利用耗散粒子动力学方法分别研究了梳状-线性共 聚物在侧链和主链选择性溶剂中的自组装行为. 在两种 溶剂环境下，该聚合物都能形成两种类型聚集体，其中 第 I 类由亲、疏溶剂链的自组装形成，而第 II 类通过线 性链与梳状亚结构间的相分离形成. 研究表明, 第 II 类 聚集体在侧链数量较多且长度较短时容易形成. 梳状线性共聚物在侧链选择性溶剂中趋于形成高曲率的结 构, 而在主链选择性溶剂中易于形成低曲率的结构. 当 该聚合物形成第 I 类聚集体，聚集体的形貌受到链段长 度的影响较大. 通过与文献报道的实验结果比较, 发现 DPD 模拟能够很好地抓住实验现象的本质.

\section{4 模拟方法}

DPD 是一种基于动量守恒和 Gallilean 不变性的介 观模拟方法. 在 DPD 中, 粒子的运动遵循牛顿运动方 程: 


$$
\frac{\mathrm{d} \boldsymbol{r}_{\alpha}}{\mathrm{d} t}=\boldsymbol{v}_{\alpha}, \quad \frac{\mathrm{d} \boldsymbol{v}_{\alpha}}{\mathrm{d} t}=\boldsymbol{f}_{\alpha}^{S}+\boldsymbol{f}_{\alpha}
$$

式中, $r_{\alpha}$ 和 $v_{\alpha}$ 分别为粒子 $\alpha$ 的位置和速度矢量; $t$ 为时 间; $\boldsymbol{f}_{\alpha}^{S}$ 是 $\alpha$ 粒子所受的键力, 而 $\boldsymbol{f}_{\alpha}$ 为除键力以外的作 用力, 包括成对的保守力 $\left(\boldsymbol{F}_{\alpha \beta}^{\mathrm{C}}\right)$ 、耗散力 $\left(\boldsymbol{F}_{\alpha \beta}^{\mathrm{D}}\right)$ 和随机力 $\left(\boldsymbol{F}_{\alpha \beta}^{\mathrm{R}}\right)$.

保守力 $\boldsymbol{F}_{\alpha \beta}^{\mathrm{C}}$ 为软作用力, 表示如下:

$$
\boldsymbol{F}_{\alpha \beta}^{\mathrm{C}}=\left\{\begin{array}{lr}
a_{\alpha \beta}\left(1-r_{\alpha \beta} / r_{c}\right) \hat{\boldsymbol{r}}_{\alpha \beta} & r_{\alpha \beta}<r_{\mathrm{c}} \\
0 & r_{\alpha \beta} \geqslant r_{\mathrm{c}}
\end{array}\right.
$$

其中, $a_{\alpha \beta}$ 为粒子 $\alpha$ 与 $\beta$ 间的最大排斥作用参数 $r_{\alpha \beta}=\left|\boldsymbol{r}_{\alpha}-\boldsymbol{r}_{\beta}\right|, \hat{\boldsymbol{r}}_{\alpha \beta}=\left(\boldsymbol{r}_{\alpha}-\boldsymbol{r}_{\beta}\right) / r_{\alpha \beta} ; \boldsymbol{r}_{\mathrm{c}}$ 为截断半径. 可见, 保守力随粒子间距离的增加而单调递减. 耗散力和随机 力的作用形式分别为

$$
\begin{aligned}
& \boldsymbol{F}_{\alpha \beta}^{\mathrm{D}}=-\gamma \omega^{\mathrm{D}}\left(r_{\alpha \beta}\right)\left(\hat{\boldsymbol{r}}_{\alpha \beta} \cdot \boldsymbol{v}_{\alpha \beta}\right) \hat{\boldsymbol{r}}_{\alpha \beta} \\
& \boldsymbol{F}_{\alpha \beta}^{\mathrm{R}}=\sigma \omega^{\mathrm{R}}\left(r_{\alpha \beta}\right) \theta_{\alpha \beta} \hat{\boldsymbol{r}}_{\alpha \beta}
\end{aligned}
$$

式中, $\gamma$ 和 $\sigma$ 分别为耗散系数和随机力系数; $\boldsymbol{v}_{\alpha \beta}=\boldsymbol{v}_{\alpha}-\boldsymbol{v}_{\beta} ; \theta_{\alpha \beta}$ 是高斯分布的随机数, 符合

$$
\begin{aligned}
& \left\langle\theta_{\alpha \beta}(t)\right\rangle=0 \\
& \left\langle\theta_{\alpha \beta}(t) \theta_{\kappa \lambda}\left(t^{\prime}\right)\right\rangle=\left(\delta_{\alpha \kappa} \delta_{\beta \lambda}+\delta_{\alpha \lambda} \delta_{\beta \kappa}\right) \delta\left(t-t^{\prime}\right)
\end{aligned}
$$

耗散力和随机力的权重函数符合下列关系:

$$
\begin{aligned}
& \omega^{\mathrm{D}}(r)=\left[\omega^{\mathrm{R}}(r)\right]^{2} \\
& \sigma^{2}=2 \gamma k_{\mathrm{B}} T
\end{aligned}
$$

式中, $k_{\mathrm{B}}$ 和 $T$ 分别为 Boltzmann 常数和温度. $\omega^{\mathrm{R}}(r)$ 取以 下形式:

$$
\omega^{\mathrm{R}}(r)= \begin{cases}1-r / r_{c} & r<r_{c} \\ 0 & r \geqslant r_{c}\end{cases}
$$

将高分子链设为珠簧模型, 键力为

$$
\boldsymbol{f}_{\alpha}^{S}=k\left(1-r_{\alpha \beta} / r_{\mathrm{eq}}\right) \hat{\boldsymbol{r}}_{\alpha \beta}
$$

其中, $k$ 和 $r_{\mathrm{eq}}$ 分别为弹性系数和平衡键长.

梳状-线性共聚物的分子模型如图 2 所示. 该分子 具有线性的 $\mathrm{A}$ 链和梳状的亚结构(包括一个主链 $\mathrm{A}$ 和多 个侧链 B). 每条链由多个粒子组成, 其中每个粒子表示 一个聚合物片段. 溶剂分子 $\mathrm{S}$ 的团簇用单个粒子表示. 模拟在 $35 \times 35 \times 35$ 的空间中进行, 体系的平均密度设 为 3 , 共聚物的浓度为 0.1 . 相同粒子之间的相互作用参 数设为 $a_{\mathrm{AA}}=a_{\mathrm{BB}}=a_{\mathrm{SS}}=25$. 此外, 亲溶剂链段与溶剂的 相互作用参数设为 25 , 而疏溶剂链段与溶剂的相互作 用参数可变. 为方便起见, 将粒子的质量 $m$ 、截断半径 $r_{\mathrm{c}}$ 和能量单位 $k_{\mathrm{B}} T$ 均设为 1.0. 弹性系数 $k$ 、耗散系数 $\gamma$ 和随机力系数 $\sigma$ 分别取 $100,4.5$ 和 3.0. 牛顿运动方程采 用 velocity-Verlet 算法求解, 时间步长取 $\Delta t=0.04 \tau$ （ $\tau=$ $\left.r_{\mathrm{c}}\left(m / k_{\mathrm{B}} T\right)^{1 / 2}\right)$. 在模拟中, 热力学平衡可通过计算热力 学量(如能量和压强)或结构参数(如均方回转半径)来判 定, 即这些参数是否随模拟时间保持不变 ${ }^{[37]}$. 事实上, 我们发现, 即使热力学量达到了稳定值(较快), 聚集体 内部结构还会变化. 因此, 将结构参数作为热力学平衡 的判据较为合理. 在研究中, 我们通过计算均方回转半 径来判定聚集体是否达到热力学平衡: 当均方回转半径 的时间平均值保持基本不变时，体系达到平衡. 在本研 究中, 模拟进行 60 万步以上，体系基本达到平衡.

\section{References}

[1] Whitesides, G. M.; Grzybowski, B. Science 2002, 295, 2418.

[2] Wang, C.; Wang, Z.; Zhang, X. Small 2011, 7, 1379.

[3] Wang, J.; Jiang, M. J. Am. Chem. Soc. 2006, 128, 3703.

[4] Mai, Y.; Zhou, Y.; Yan, D. Small 2007, 3, 1170.

[5] Li, G.; Shi, L.; Ma, R.; An, Y.; Huang, N. Angew. Chem., Int. Ed. 2006, 118,5081 .

[6] Cai, C.; Lin, J.; Chen, T.; Wang, X.; Lin, S. Chem. Commun. 2009, 2709.

[7] Fan, J.; Han, Y.; Jiang, W. Acta Chim. Sinica 2011, 69, 2341. (樊娟 娟, 韩媛媛, 姜伟, 化学学报, 2011, 69, 2341.)

[8] Yang, Y.; Sun, Q.; Zhang, C.; Guo, X.; Zhang, L.; Wen, X. Acta Chim. Sinica 2012, 70, 505. (杨友强, 孙清清, 张灿阳, 郭新东, 章莉娟, 文秀芳, 化学学报, 2012, 70, 505.)

[9] Li, Z.; Qian, J.; Cao, X.; Song, X.; Wu, F. Acta Chim. Sinica 2010, 68,181 . (李振泉, 钱健, 曹绪龙, 宋新旺, 吴飞鹏, 化学学报, 2010, 68, 181.)

[10] Zhang, L.; Eisenberg, A. Science 1995, 268, 1728.

[11] Discher, D. E.; Eisenberg, A. Science 2002, 297, 967.

[12] Nap, R. J.; ten Brinke, G. Macromolecules 2002, 35, 952.

[13] Nap, R. J.; Kok, C.; ten Brinke, G.; Kuchanov, S. I. Eur. Phys. J. E 2001, 4, 515.

[14] Wang, L.; Zhang, L.; Lin, J. J. Chem. Phys. 2008, 129, 114905.

[15] Wang, L.; Lin, J.; Zhang, L. Langmuir 2009, 25, 4735.

[16] Chiang, W. S.; Lin, C. H.; Yeh, C. L.; Nandan, B.; Hsu, P. N.; Lin, C. W.; Chen, H. L.; Chen, W. C. Macromolecules 2009, 42, 2304.

[17] Huang, C. I.; Lin, Y. H. Macromol. Rapid Commum. 2007, 28, 1634

[18] Xu, F.; Li, T.; Xia, J.; Qiu, F.; Yang, Y. Polymer 2007, 48, 1428.

[19] Neiser, M. W.; Muth, S.; Kolb, U.; Harris, J. R.; Okuda, J.; Schmidt, M. Angew Chem., Int. Ed. 2004, 43, 3192

[20] Khelfallah, N.; Gunari, N.; Fisher, K.; Gkogkas, G.; Hadjichristidis, N.; Schmidt, M. Macromol. Rapid Commun. 2005, 26, 1693.

[21] Peng, S.; Bhushan, B. RSC Adv. 2012, 2, 8557.

[22] Luo, Y.; Liu, L.; Wang, X.; Shi, H.; Lv, W.; Li, J. Soft Matter 2012, $8,1634$.

[23] Du, J.; Chen, D.; Wang, Y.; Xiao, C.; Lu, Y.; Wang, J.; Zhang, G. Biomacromolecules 2006, 7, 1898.

[24] Bao, R.; Li, L.; Qiu, F.; Yang, Y. Acta Chim. Sinica 2011, 69, 2511. (鲍稔, 李莉, 邱枫, 杨玉良, 化学学报, 2011, 69, 2511.)

[25] Wang, J.; Guo, K.; An, L.; Müller, M.; Wang, Z.-G. Macromolecules 2010, 43, 2037.

[26] Koelman, J. M. V. A.; Hoogerbrugge, P. J. Europhys. Lett. 1993, 21, 363.

[27] Hoogerbrugge, P. J.; Koelman, J. M. V. A. Europhys. Lett. 1992, 19, 155.

[28] Groot, R. D.; Madden, T. J.; Tildesley, D. J. J. Chem. Phys. 1999, $108,9737$.

[29] Groot, R. D.; Warren, P. B. J. Chem. Phys. 1997, 107, 4423.

[30] Li, X.; Pivkin, I. V.; Liang, H.; Karniadakis, G. E. Macromolecules 2009, 42, 3195 .

[31] Xin, J.; Liu, D.; Zhong, C. J. Phys. Chem. B 2007, 111, 13675.

[32] Zhao, Y.; Liu, Y.-T.; Lu, Z.-Y.; Sun, C.-C. Polymer 2008, 49, 4899.

[33] Yu, Y.; Feng, J.; Liu, H.; Hu, Y. Mol. Simulat. 2008, 34, 559. 
[34] Jiang, T.; Wang, L.; Lin, S.; Lin, J.; Li, Y. Langmuir 2011, 27, 6440.

[35] Liu, H.; Guo, H.; Zhou, J. Acta Chim. Sinica 2012, 70, 2445. (刘红 艳, 郭泓雨, 周健, 化学学报, 2012, 70, 2445.)

[36] Lin, S.; Numasawa, N.; Nose, T.; Lin, J. Macromolecules 2007, 40,
1684.

[37] Rapaport, D. C. The Art of Molecular Dynamics Simulation, Cambridge University Press, Cambridge, 1995.

(Zhao, X.) 